\title{
The PAH emission spectra of Large Magellanic Cloud H II regions $^{\star}$
}

\author{
R. Vermeij ${ }^{1}$, E. Peeters ${ }^{2,1}$, A. G. G. M. Tielens ${ }^{1,2}$, and J. M. van der Hulst ${ }^{1}$ \\ 1 Kapteyn Astronomical Institute, PO Box 800, 9700 AV Groningen, The Netherlands \\ 2 SRON National Institute for Space Research, PO Box 800, 9700 AV Groningen, The Netherlands
}

Received 11 September 2001 / Accepted 8 November 2001

\begin{abstract}
A set of ISOPHOT spectra from a sample of Hin regions in the Large Magellanic Cloud (LMC) is presented. In all the spectra, emission bands arising from Polycyclic Aromatic Hydrocarbons (PAHs) are clearly present. These features are observed to vary considerably in relative strength to each other from source to source and even within 30 Doradus. The LMC spectra have been compared with ISO-SWS spectra from Galactic H II regions and with the ISOCAM observation towards a quiescent molecular cloud in the SMC (Reach et al. 2000). A correlation is found between the $I_{7.7} / I_{11.2}$ versus $I_{6.2} / I_{11.2}$ and the $I_{8.6} / I_{11.2}$ versus $I_{6.2} / I_{11.2}$ ratios. A segregation between the sources in the different types of environment (Milky Way - LMC - SMC) is present. Furthermore, within the LMC observations, a clear distinction between 30 Doradus and non-30 Doradus pointings is found. We discuss the variations in the relative strength of the PAH features in view of the different physical environments and highlight the relation with the $\mathrm{PAH} /$ dust ratio and the extinction curve. We conclude that 1) the same conditions responsible for the observed trends in the relative PAH-feature strengths also affect the carrier of the $2175 \AA$ bump leading to the differences in strength of the latter, and 2) the molecular structure is the major cause of the observed variations in the relative strength of the PAH features. In the SMC and 30 Doradus compact PAH species dominate, while PAHs with an open, uneven structure are the dominant ones in Galactic H II regions and the non-30 Dor LMC sources.
\end{abstract}

Key words. infrared: ISM: lines and bands - ISM: molecules - H II regions - Magellanic Clouds

\section{Introduction}

Ground-based, airborne, and space-based observations have shown that the mid-infrared spectra of many objects with associated dust and gas are dominated by the wellknown emission features at 3.3, 6.2, 7.7, 8.6 and $11.2 \mu \mathrm{m}$ commonly called the unidentified infrared (UIR) bands (cf. Gillett et al. 1973; Geballe et al. 1985; Cohen et al. 1986; Roche et al. 1989). These bands are now generally attributed to vibrational relaxation of UV-pumped Polycyclic Aromatic Hydrocarbons (PAHs) containing $\simeq 50$ carbon atoms (Léger \& Puget 1984; Allamandola et al. 1985, 1989; Puget \& Léger 1989; Tielens et al. 2000). The band at $3.3 \mu \mathrm{m}$ is due to $\mathrm{CH}$ stretching vibrations, the $6.2 \mu \mathrm{m}$ band corresponds largely to pure CC stretching motions, whereas the $7.7 \mu \mathrm{m}$ feature arises from combinations of $\mathrm{CC}$ stretching and $\mathrm{CH}$ in-plane bending motions. The 8.6 and $11.2 \mu \mathrm{m}$ features arise from $\mathrm{CH}$ in-plane and out-of-plane bending motions respectively. Underlying the

\footnotetext{
* Based on observations with ISO, an ESA project with instruments funded by ESA Member States (especially the PI countries: France, Germany, The Netherlands and the UK) and with the participation of ISAS and NASA.
}

$6.2 \mu \mathrm{m}, 7.7 \mu \mathrm{m}$ and $8.6 \mu \mathrm{m}$ bands as well as underlying the $11.2 \mu \mathrm{m}$ band, is a quasi-continuum or pedestal originating from larger PAHs and clusters of small PAHs.

Since the emission arises not from a single species but from a complex mixture of PAHs (Léger \& Puget 1984; Allamandola et al. 1985), the interstellar PAH spectra reflect a combination of individual molecular features, and hence the overall PAH spectrum appears very similar from source to source. The exact PAH family (molecular structure and size) present in a source fine-tunes the PAH spectrum in terms of relative strengths and peak positions of the individual PAH features (Schutte et al. 1993; Verstraete et al. 1996; Allamandola et al. 1999; Moutou et al. 1999; Hony et al 2001; Peeters et al. 2001b). Laboratory studies and quantum-chemical calculations have shown that ionization of the PAH molecules has a remarkable effect on the spectrum. While the peak positions of the UIR bands are modestly affected by ionization, the influence on the intensity is striking - particularly in the 5 to $10 \mu \mathrm{m}$ region (Allamandola et al. 1999; Bakes et al. 2001). As a result, the emitted spectrum will depend on the local physical conditions. In addition, dehydrogenation may also influence the PAH spectrum, in 
particular in the strength of the different $\mathrm{CH}$ modes (Pauzat et al. 1997).

With the launch of the Infrared Space Observatory (ISO), a huge amount of infrared spectral data became available, showing the omnipresent nature of these PAH features. By now, the features have been detected in a wide range of objects and environments, from post-AGB stars and planetary nebulae, to $\mathrm{H}$ II regions, reflection nebulae, the diffuse interstellar medium and extragalactic sources. As demonstrated by the galaxy survey by Helou (1999), the mid-IR spectrum of the ISM in galaxies is dominated by the UIR bands. A spectroscopic survey of the nuclei of nearby $(z<0.3)$ starburst galaxies has also revealed the presence of bright UIR bands (Bosselli et al. 1997; Lutz et al. 1998).

Studies have also been performed for the Magellanic Clouds. ISOCAM studies of the large SMC H II region N 66 (Contursi et al. 2000) and of the SMC molecular cloud B1\#1 (Reach et al. 2000) show the presence of PAHs even in such a metal-deficient environment. A similar study of the H II region complex N4 in the LMC also hints at the presence of PAHs (Contursi et al. 1998). An ISO-SWS AOT1 spectrum of 30 Doradus taken at our position \#3 also shows the clear presence of PAHs (Sturm et al. 2000).

The study of PAHs in the Magellanic Clouds is of interest because of the different physical environment encountered there. The best known of these differences is the low-metallicity of the Magellanic Clouds relative to the Galaxy. Furthermore, the prodigious starformation activity inundates the Magellanic Clouds with diffuse UV radiation (e.g. Cole et al. 1999).

In this paper, a set of mid-IR spectra from a sample of $\mathrm{H}$ II regions in the Large Magellanic Cloud is presented, and all of these show the strong presence of PAH features. These spectra give us the opportunity to investigate the influence of the completely different environment in the LMC on the PAH spectra and their carriers. We therefore make a comparison between our sample $\mathrm{H}$ II regions in the LMC and those of our own Milky Way and the SMC. In Sect. 2 the data are presented, with special emphasis on the way the PAH-feature strengths have been determined. Section 3 describes the general trends in the observed PAH-feature strengths. In Sect. 4, the connection with extinction is highlighted and the possible underlying causes for the observed trends are examined. Finally, Sect. 5 summarises our results.

\section{The data}

\subsection{Observations}

The sample includes a set of Large Magellanic Cloud $\mathrm{H}$ II regions. The spectra were obtained with the ISOPHOT/PHT-S instrument on board ISO in staring mode using the PHT-40 template. The observation log is given in Table 1. The PHT-S instrument covers the spectral ranges from $2.5-4.8 \mu \mathrm{m}$ (the PHT-SS part) and from 5.8-11.6 $\mu \mathrm{m}$ (the PHT-SL part). Both spectral ranges

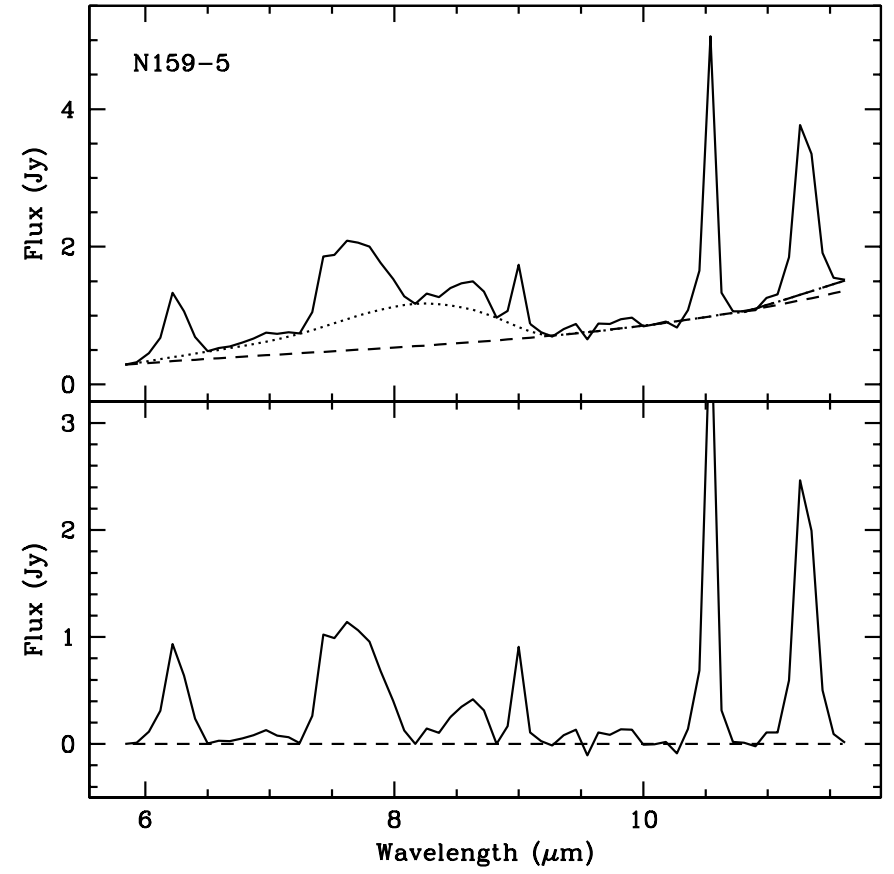

Fig. 1. The ISOPHOT spectrum of N159-5. The upper panel shows the spectrum and the components of the fitted continuum. The lower panel shows the continuum-subtracted spectrum.

have their own linear detector block consisting of 64 pixels each with a spectral resolution of 85 in the SS part and 95 in the SL part. The ISOPHOT spectra were reduced with PIA V8.4 using standard routines. Special attention is paid to the de-glitching of the signal from every separate pixel. This is done to make sure that no spurious feature would contaminate the spectra. In addition to these ISOPHOT data, the ISO-SWS AOT 01 spectrum of 30 Dor\#3 (Sturm et al. 2000) was taken from the ISO archive and reduced in a similar way as the spectra of the Galactic H II regions. Representing the Small Magellanic Cloud in this work is the ISOCAM observation from Reach et al. (2000) of the molecular cloud B1\#1.

The sample of H II regions in the Milky Way was taken from the Galactic "Ultra Compact H II region" ISO program (Peeters et al. 2001a) complemented with spectra of the Orionbar also taken from the ISO archive (Orion bar\#3: Cesarsky et al. 2000). The spectra have been obtained with the Short Wavelength Spectrometer (SWS) in the AOT01/AOT06 scanning mode (see Table 1). A detailed account of the reduction of the AOT 01 spectra can be found in Peeters et al. (2001a). The AOT 06 spectra were reduced in a similar way as the AOT 01 data.

\subsection{The spectra}

The ISOPHOT spectra of the LMC sources are shown in the Figs. 1 and 2. Only the part of the spectra ranging from 5.9 to $11.6 \mu \mathrm{m}$ is shown here. The spectra of the LMC HII regions resemble the spectra of Galactic H II regions very much. All the major $\mathrm{PAH}$ features, i.e. the 
Table 1. The ISOPHOT and ISO-SWS pointings and dates of the observations. The coordinates are J2000.0. The designations for the Magellanic Cloud sources, except for 30 Doradus, are those of Henize (1956). The Galactic sources (except the Orionbar) are designated by their IRAS number.

\begin{tabular}{|c|c|c|c|c|c|c|c|c|}
\hline \multirow[b]{2}{*}{ Object } & \multirow[b]{2}{*}{$\mathrm{RA}(\mathrm{h} \mathrm{m} \mathrm{s})$} & \multirow{2}{*}{\multicolumn{2}{|c|}{ LMC $\overline{\delta\left({ }^{\circ}, \prime^{\prime \prime}\right)}$}} & \multirow[b]{2}{*}{ Object } & \multicolumn{2}{|c|}{ Milky Way } & \multirow[b]{2}{*}{ Date } & \multirow[b]{2}{*}{$\mathrm{AOT}$} \\
\hline & & & & & $\mathrm{RA}(\mathrm{h} \mathrm{m} \mathrm{s})$ & $\delta\left(\begin{array}{lll}\mathrm{o} & \prime & \prime \prime\end{array}\right)$ & & \\
\hline $\mathrm{N} 4 \mathrm{~A}$ & 45208.4 & -665523.4 & 29-Apr.-96 & Orionbar\#1 & 053521.4 & -052540.1 & 11-Oct.-97 & 01 \\
\hline N83B & 45425.8 & -691056.7 & 31-Oct.-97 & Orionbar\#2 & 053519.8 & -052510.0 & 24-Feb.-98 & 01 \\
\hline N11A & 45716.3 & -662322.9 & 16-Aug.-96 & Orionbar\#3 & 053518.2 & -052439.9 & 11-Oct.-97 & 01 \\
\hline N159-5 & $\begin{array}{lll}5 & 40 & 04.2\end{array}$ & -694442.9 & 24-Sep.-97 & $12331-6134$ & 123601.9 & -615103.9 & 10-Sep.-96 & 01 \\
\hline 30 Dor \#1 & 53833.5 & -690627.1 & 05-Dec.-97 & $15384-5348$ & 154217.1 & -535831.5 & 10-Sep.-96 & 01 \\
\hline 30 Dor \#2 & 53835.4 & -690541.2 & 13-Apr.-96 & $18317-0757$ & 183424.9 & -075447.9 & 08-Mar.-97 & 01 \\
\hline 30 Dor \#3 & 53846.0 & -690507.9 & 20-Jul.-97 & $18434-0242$ & 184804.1 & -023920.0 & 12-Apr.-97 & 06 \\
\hline \multirow[t]{4}{*}{30 Dor \#4 } & 53854.2 & $\begin{array}{lll}-69 & 05 & 15.3\end{array}$ & 05-Aug.-97 & $21190+5140$ & 212044.9 & +515327.0 & 30-Nov.-97 & 06 \\
\hline & & & & $22308+5812$ & 223245.9 & +582821.0 & 12-May-96 & 01 \\
\hline & & & & $23030+5958$ & 230510.6 & +501441.0 & 06-Dec.-97 & 06 \\
\hline & & & & $23133+6050$ & 231531.4 & +510708.0 & 06-Jun.-97 & 06 \\
\hline
\end{tabular}
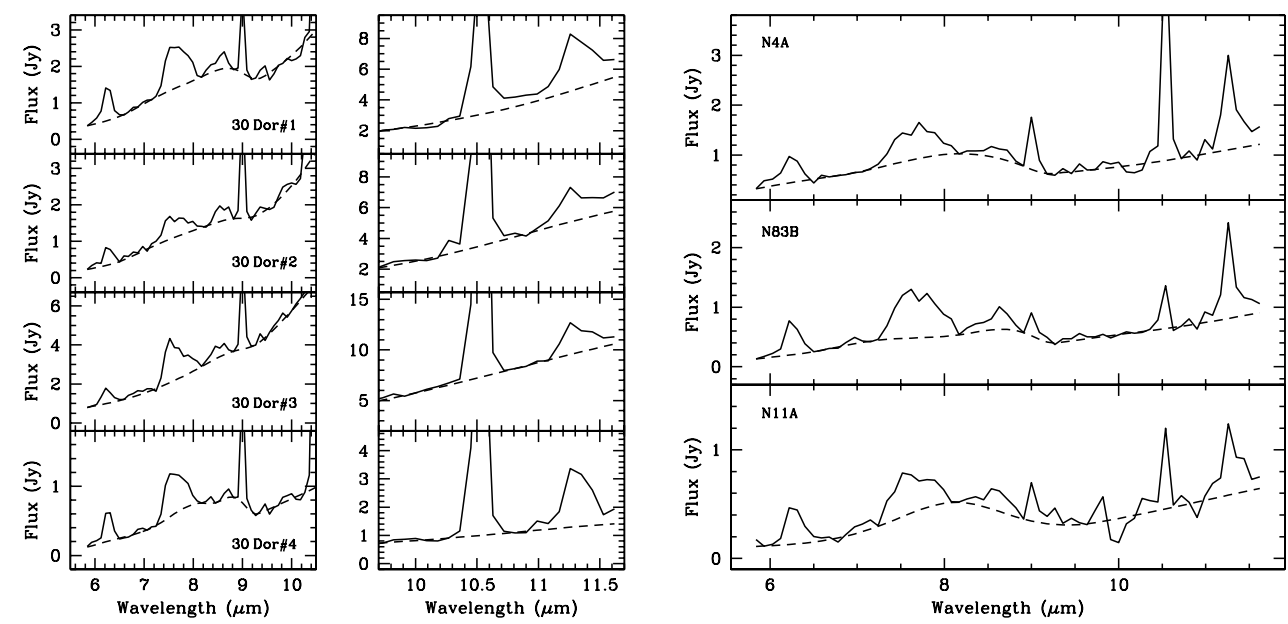

Fig. 2. The ISOPHOT spectra of the sample objects in the Large Magellanic Cloud. The left panel shows the 30 Doradus spectra from which the spectral region near $11 \mu \mathrm{m}$ is blown up for clarity.

$6.2,7.7,8.6$ and $11.2 \mu \mathrm{m}$ bands, are clearly present. Only in some cases (e.g. N4A), a trace of the $3.3 \mu \mathrm{m}$ feature was seen. This is not surprising since this feature is known to contribute typically only $6 \%$ to the total luminosity carried by the PAH features (Cohen et al. 1989) and can therefore easily be hidden in the noise in the ISOPHOT spectra. Even the weak PAH features at 6.0 and $11.0 \mu \mathrm{m}$ (Hony et al. 2001; Peeters et al. 2001b) can be discerned in some of the spectra (e.g. N4A, 30Dor\#4). In addition to the PAH emission bands, the [Ar II] $6.9 \mu \mathrm{m}$, [Ar III] $8.9 \mu \mathrm{m}$ and [S IV] $10.5 \mu \mathrm{m}$ fine-structure lines are observed, the former being rather weak. The Pf $\alpha$ line at $7.5 \mu \mathrm{m}$ might be present in the spectra, but at the resolution of PHT-S it is not possible to disentangle the Pf $\alpha$ line from the $7.7 \mu \mathrm{m}$ $\mathrm{PAH}$ feature. This line and the PAH feature are easily separated at the resolution of SWS. In the SWS spectrum of 30 Dor\#3, this $\mathrm{Pf} \alpha$ line is clearly present. The silicate absorption feature at $9.7 \mu \mathrm{m}$ was not detected in any of the spectra of the Magellanic sources.

Already at first glance, variations in the relative PAHfeature strengths from one object to another are obvious.
These variations are not only present when comparing different $\mathrm{H}$ II regions, but also for the different pointings within the giant $\mathrm{H}$ II region 30 Doradus. At first sight, the shape of the features also seems to vary. This is the most pronounced for the $7.7 \mu \mathrm{m}$ feature, but here the possible presence of the Pf $\alpha$ line at $7.5 \mu \mathrm{m}$ should be taken into account. While tantalizing, we conclude that these profile variations may not be significant due to the low resolution of the PHT-S instrument. Note, however, that the Galactic H II regions show little profile variations for the 6.2, 7.7 and $8.6 \mu \mathrm{m}$ features (see Peeters et al. 2001b). Small variations are present in the $11.2 \mu \mathrm{m}$ PAH feature (see Peeters et al. 2002, in prep.) but these cannot be seen at the PHT-S resolution. Furthermore, the PAH profiles in extragalactic sources are similar to those in the Galactic H II regions (see Peeters et al. 2001b).

\subsection{Continuum fitting and flux determination}

For both the ISOPHOT and the ISO-SWS spectra the continuum-subtraction was done with the same two-step 
procedure by fitting a spline through selected points in the spectra.

In the first step of the continuum-subtraction, an underlying dust continuum was defined. For the ISOPHOT spectra, the spline points for this continuum were located at $\sim 5.8 \mu \mathrm{m}$, in the $9-10 \mu \mathrm{m}$ region, and at the blue base of the $[\mathrm{SIV}] 10.5 \mu \mathrm{m}$ line. The wavelength coverage of ISOPHOT (ending at $11.6 \mu \mathrm{m}$ ) hampers the determination of the continuum redwards of the [S IV] $10.5 \mu \mathrm{m}$ line. Without continuum points beyond $11.6 \mu \mathrm{m}$, it is difficult to ascertain the presence of a broad plateau underlying the $11.2 \mu \mathrm{m}$ feature and, if present, to distinguish it from the underlying dust continuum. Therefore, no points were added in this region to constrain the fit to the underlying continuum. However, as can be seen in, for example, Fig. 1, some residual flux is present underneath the $11.2 \mu \mathrm{m}$ feature. In the spectra where such a residual flux was present, it was removed by defining a simple straight-line continuum superimposed on the underlying continuum.

For $\mathrm{H}$ II regions, the $5-9 \mu \mathrm{m}$ spectral range is also characterized by a broad plateau underlying the features in this range (Peeters et al. 2001b). In the second step, a continuum was fit to this plateau and subtracted. Anchor points for the fit to this plateau were located at $\sim 5.8 \mu \mathrm{m}$, between the $6.2 \mu \mathrm{m}$ and the $7.7 \mu \mathrm{m}$ features, at the blendpoint of the $7.7 \mu \mathrm{m}$ and $8.6 \mu \mathrm{m}$ features, and at the base of the [Ar III] $8.9 \mu \mathrm{m}$ line. As an example, the resulting composite continuum and the continuum-subtracted spectrum of N159-5 are shown in Fig. 1.

For fitting the underlying continuum in the SWS spectra, points in roughly the same wavelength ranges were taken. A spline was fitted through points in the range from $5-6 \mu \mathrm{m}$, between the $6.2 \mu \mathrm{m}$ and the $7.7 \mu \mathrm{m}$ features (near $7 \mu \mathrm{m}$ ), between the $7.7 \mu \mathrm{m}$ and $8.6 \mu \mathrm{m}$ features (near $8.2 \mu \mathrm{m}$ ), in the $9-11 \mu \mathrm{m}$ range and through points near $11.7 \mu \mathrm{m}$.

In this way, a local continuum around the $6.2 \mu \mathrm{m}$ and the $11.2 \mu \mathrm{m}$ features is determined. The presence of a silicate absorption feature at $9.7 \mu \mathrm{m}$ (in some Galactic H II regions) has been ignored in determining the fit but will strongly influence the $8.6 \mu \mathrm{m}$ feature. Therefore, when examing the $8.6 \mu \mathrm{m}$ feature, the Galactic H II regions with obvious silicate absorption (IRAS 15384, IRAS 18317, IRAS 18434) are disregarded.

The fluxes of the PAH features have been derived from the continuum-subtracted spectra by simple pixel integration. The fluxes of the $6.2 \mu \mathrm{m}, 7.7 \mu \mathrm{m}, 8.6 \mu \mathrm{m}$ and $11.2 \mu \mathrm{m}$ features were derived by integration over the wavelength intervals of $6.1-6.6 \mu \mathrm{m}, 7.1-8.2 \mu \mathrm{m}, 8.3-8.9 \mu \mathrm{m}$ and $11.1-$ $11.6 \mu \mathrm{m}$, respectively. To make sure that the fluxes for the SMC B1\#1 cloud can be compared, these have been rederived in the same way rather than taking the fluxes from Reach et al. (2000). The fluxes will be sensitive to the continuum determination and the decomposition of the 6 to $9 \mu \mathrm{m}$ region in terms of the $\mathrm{PAH}$ features and the PAH plateau. However, this will influence all spectra in the same way. In particular, this will not introduce spurious differences between the Galactic and the LMC sources.

The fluxes for the LMC and SMC sources are given in Table 2. For 30 Doradus \#3, both the flux derived from the ISOPHOT data as well as the SWS data is given in Table 2. The only uncertainty quoted in the table is the one arising from noise in the spectra. The relative flux calibration uncertainty of the ISOPHOT spectra is estimated to be around $10 \%$ (Acosta-Pulido \& Ábrahám 2001). The $7.7 \mu \mathrm{m}$ flux could be contaminated by the Pf $\alpha$ line, but a comparison between the $7.7 \mu \mathrm{m}$ fluxes and the Pf $\alpha$ fluxes for the same objects from Vermeij et al. (2001) showed this contamination to be not higher than $\sim 10 \%$. For the Galactic H II regions, the intensity of the Pf $\alpha$ line is less then $8 \%$ of the intensity of the $7.7 \mu \mathrm{m}$ PAH band.

\section{Correlations}

Given the differences in the physical environments of the Milky Way and the Magellanic Clouds, it is of interest to investigate the differences - if any - in the PAH spectra between Galactic and Magellanic Cloud H II regions. Here we want to study variations in the relative strength of the $\mathrm{PAH}$ bands to each other, not the differences in absolute intensities due to intrinsic luminosity and distance of the sources. We therefore use 3 -feature intensity ratio plots. The relation between the different $\mathrm{PAH}$-feature strengths is shown in Fig. 3 .

Three of our Galactic sources suffer from extinction (IRAS 15384, IRAS 18317, IRAS 18434; MartínHernández et al. 2001). For these sources, both the observed and intrinsic ratios are shown in the left panel of Fig. 3 (the dashed arrows). The extinction $A_{\mathrm{K}}$ is derived from hydrogen recombination lines. These sources are not shown in the right panel of Fig. 3 since silicate absorption influences the relative strength of the $8.6 \mu \mathrm{m}$ feature. For the LMC sources, the pointing with the highest extinction (30 Dor\#4) has an $A_{V}$ of 1.7 (Vermeij et al. 2001). Therefore, extinction only has a minor influence on the observed strengths of the PAH features in the LMC. Both panels clearly show linear correlations between the different PAH-feature strengths. Most striking is a segregation between the sources of different type of environment, i.e. from the Milky Way to the LMC and to the SMC. Interesting, however, is the fact that for 30 Doradus the measured PAH-feature ratios lie further down the sequence than those of the other LMC pointings, showing a clear distinction between the pointings in 30 Doradus and the non-30 Doradus sources. This distinction is clearly visible for the $I_{6.2} / I_{11.2}$ ratio, but is much less pronounced for the $I_{7.7} / I_{11.2}$ and the $I_{8.6} / I_{11.2}$ ratios (Fig. 3). Three of the pointings in 30 Doradus show a $I_{7.7} / I_{11.2}$ and $I_{8.6} / I_{11.2}$ ratio similar to those of the Galactic and non-30 Dor sources. Note that the non-30 Dor LMC H II regions behave more like the Galactic H II regions in our sample.

We also investigated the relative contribution of every PAH feature to the total emitted PAH flux in all the features. These are shown in Fig. 4. The SMC point is 
Table 2. The ISOPHOT fluxes of the different PAH features in units of $10^{-18} \mathrm{~W} \mathrm{~cm}^{-2}$. The errors given in this table are only the measurement errors. Also included are the fluxes from SMC B1\#1 and the SWS-AOT1 spectrum of 30 Doradus\#3.

\begin{tabular}{lrrrrr}
\hline Object & $3.3 \mu \mathrm{m}$ & $6.2 \mu \mathrm{m}$ & $7.7 \mu \mathrm{m}$ & $8.6 \mu \mathrm{m}$ & $11.2 \mu \mathrm{m}$ \\
\hline N4A & $\mathrm{d}$ & $0.92 \pm 0.05$ & $1.67 \pm 0.02$ & $0.29 \pm 0.01$ & $0.68 \pm 0.03$ \\
N83B & $\mathrm{n}$ & $0.85 \pm 0.01$ & $2.05 \pm 0.02$ & $0.46 \pm 0.04$ & $0.56 \pm 0.02$ \\
N11A & $\mathrm{n}$ & $0.60 \pm 0.01$ & $0.92 \pm 0.02$ & $0.26 \pm 0.01$ & $0.29 \pm 0.01$ \\
N159-5 & $\mathrm{n}$ & $1.45 \pm 0.02$ & $2.83 \pm 0.02$ & $0.54 \pm 0.05$ & $1.14 \pm 0.01$ \\
30 Dor\#1 & $\mathrm{d}$ & $1.59 \pm 0.03$ & $2.87 \pm 0.02$ & $0.51 \pm 0.01$ & $1.48 \pm 0.01$ \\
30 Dor\#2 & $\mathrm{n}$ & $0.79 \pm 0.05$ & $1.50 \pm 0.03$ & $0.48 \pm 0.01$ & $0.92 \pm 0.02$ \\
30 Dor\#3 & $\mathrm{d}$ & $1.27 \pm 0.02$ & $4.50 \pm 0.04$ & $0.81 \pm 0.01$ & $1.51 \pm 0.02$ \\
30 Dor\#3 & 0.23 & 1.16 & 3.66 & 0.74 & 1.23 \\
30 Dor\#4 & $\mathrm{n}$ & $0.65 \pm 0.02$ & $1.38 \pm 0.02$ & $0.11 \pm 0.05$ & $1.07 \pm 0.04$ \\
SMC B1\#1 $^{\dagger}$ & - & 0.37 & 0.76 & 0.11 & 1.26 \\
\hline
\end{tabular}

${ }^{\circ}$ : Flux derived from the ISOPHOT data. ${ }^{*}$ : Flux derived from the ISO-SWS AOT 01 spectrum (Sturm et al. 2000).

${ }^{\dagger}$ : Flux derived from the ISOCAM spectrum (Reach et al. 2000), see Sect. 2.3. n: Not detected. d: Detected.
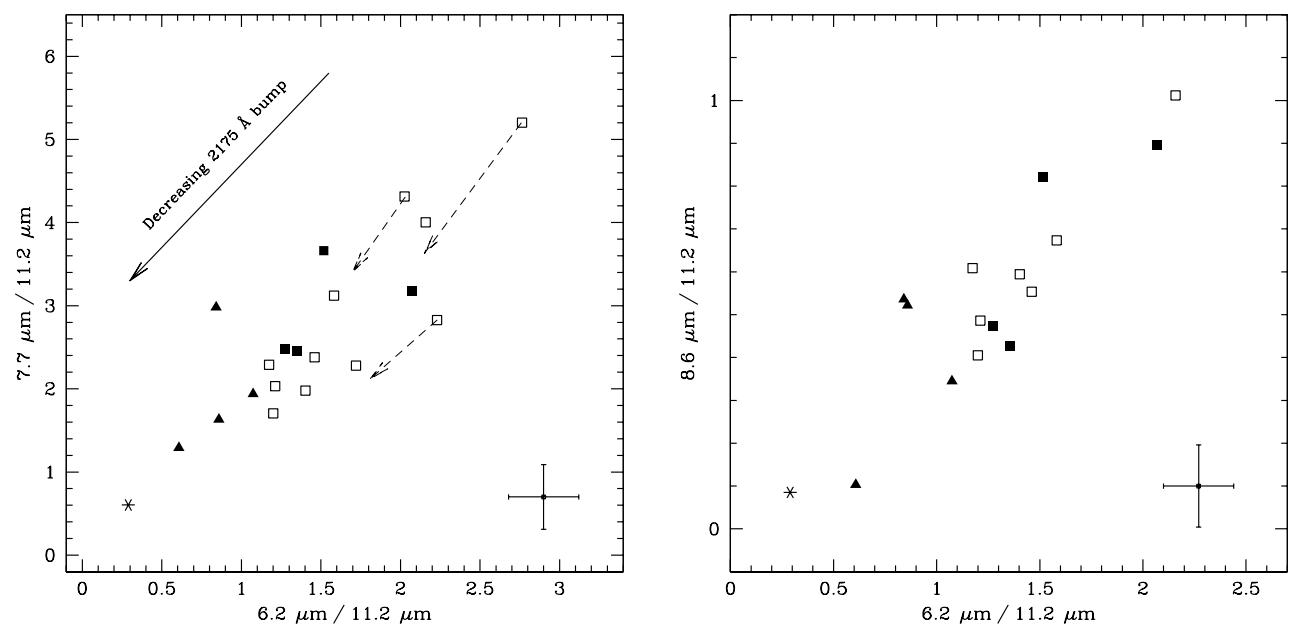

Fig. 3. The correlations between the different relative PAH-feature strengths. The feature fluxes have been normalized relative to the $11.2 \mu \mathrm{m}$ flux so as to eliminate the differences in the distance to the source. The Galactic sources are showns as open squares, the non-30 Dor sources as solid squares and the 30 Dor pointings as solid triangles. The asterisk represent the SMC B1\#1 molecular cloud. Shown in the lower right corner is a typical error bar. The dashed arrows shows the shift in position of the observed ratios when an extinction correction is applied (see text). The solid arrow shows the direction in which the contrast of the $2175 \AA$ bump to the general extinction decreases.
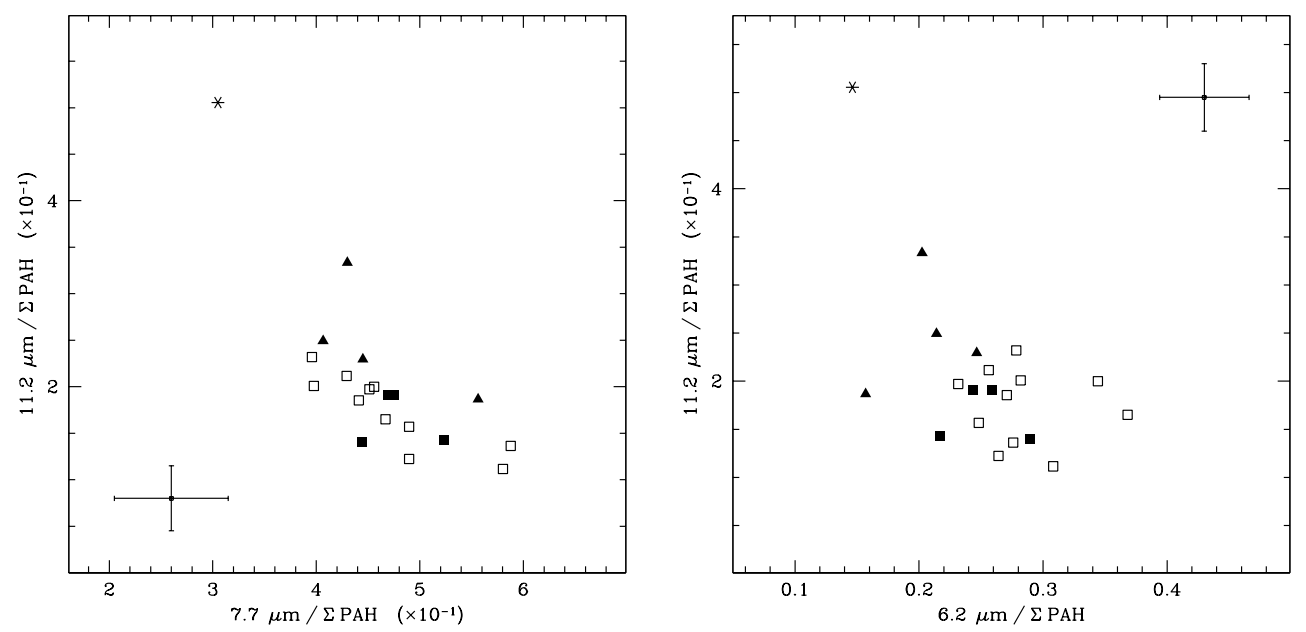

Fig. 4. The relative contribution of the $6.2,7.7$ and $11.2 \mu \mathrm{m}$ PAH features to the total energy output within all the PAH bands. For an explanation of the symbols see Fig. 3. Shown in both panels is a typical errorbar. 
in all the plots clearly separated from the other objects. The Galactic and Magellanic Cloud sources show a similar range in the fraction of the PAH emission emitted in the $7.7 \mu \mathrm{m}$ and the $8.6 \mu \mathrm{m}$ band. In contrast, there is a clear difference between the Galactic sources and the 30 Dor and SMC sources when considering the 6.2 and $11.2 \mu \mathrm{m}$ bands (Fig. 4, right panel). The $6.2 \mu \mathrm{m}$ feature is relatively stronger in the Galactic sources whereas its $11.2 \mu \mathrm{m}$ band is relatively weaker. This latter difference, however, is only slight. Here too, the 30 Dor pointings and the SMC source are situated in different regions in the plot compared to the Galactic sources and the non-30 Dor sources. A similar separation is not noticed for the $I_{7.7} / \Sigma \mathrm{PAH}$ versus $I_{11.2} / \Sigma$ PAH ratio (Fig. 4, left panel).

\section{Discussion}

\subsection{Extinction: The $2175 \AA$ bump}

Much work has been done on determining the extinction characteristics of many lines-of-sight towards the LMC and also the SMC (e.g. Borgman et al. 1975; Nandy et al. 1981; Koornneef \& Code 1981; Prévot et al. 1984). A common result found in this work for the LMC is that the extinction in the UV is significantly higher and rises more sharply than for Galactic lines-of-sight and that the $2175 \AA$ bump is about $50 \%$ weaker. This change in the characteristics of the extinction curve is even more pronounced for the SMC. It has also been pointed out that there seems to be an anti-correlation between the $2175 \AA$ bump strength and the slope of the FUV rise.

For a long time, it was assumed that the extinction curve derived for the LMC was applicable for the galaxy as a whole. However, this early work was based solely on stars near 30 Doradus. Later work by Fitzpatrick (1985, 1986) and Clayton \& Martin (1985) showed the extinction curve outside the 30 Dor region to be much less different from the Galactic ones than previously thought, with the $2175 \AA$ feature significantly stronger than in 30 Dor. The carrier of the $2175 \AA$ bump has not yet been positively identified, but one of the possible candidates could be PAHs (Joblin et al. 1992).

Our pointings are nicely distributed over the LMC and can be divided into a 30 Dor group and a non-30 Dor group. Some of the stars used by Fitzpatrick (1986) in the derivation of his non-30 Dor curves are located near our sample objects N11A, N4A and N83B (e.g. Sk 19-66, Sk 267, Sk 19-69). It is therefore interesting to compare our PAH spectra with the properties of the extinction curves.

A comparison between the absolute PAH-feature fluxes for our 30 Dor pointings and for our other LMC objects does not show many differences (see Table 2). The relative intensity ratios though show a clear sequence, going from the Galaxy and the non-30 Dor pointings to 30 Dor and the SMC object B1\#1 (see Fig. 3). A similar sequence can be found for the $2175 \AA$ bump strength, which decreases in strength going from the Milky Way to the non-30 Dor and the 30 Dor pointings and is even completely absent in the
SMC (e.g. Rodrigues et al. 1997). The latter suggests a possible connection between the strength of the $2175 \AA$ bump on one hand and the PAHs and their structure on the other hand. The direction in which the contrast of the $2175 \AA$ feature decreases relative to the general extinction is shown in Fig. 3 .

The confirmation or refutation of PAHs as carrier of the $2175 \AA$ bump is difficult though. Extinction curves are commonly derived from lines-of-sight towards hot stars, and it is not easy to asses the impact of the environment on the molecules. Moreover, the possibility exists that the same physical conditions responsible for our observed trends in the relative PAH-feature strengths also affect the carrier of the $2175 \AA$ bump leading to the differences in strength of the latter. To really settle the problem of the possible PAH nature of the bump carrier, observations of PAHs and extinction curves in the same line-ofsight are needed. In the next section, we will discuss several processes affecting the strength of the different $\mathrm{PAH}$ features.

\subsection{Variations in the observed PAH spectrum}

Many different factors play a role in shaping the $\mathrm{PAH}$ spectra and their relative PAH-feature strengths. Among these are the balance between ionized and neutral PAHs, the degree of hydrogenation of the PAHs, and the size and molecular structure of the PAHs present in the source (e.g. Schutte et al. 1993; Allamandola et al. 1999; Hudgins \& Allamandola 1999; Bakes et al. 2001). The problem one faces when analyzing a PAH spectrum is the fact that all these possible factors are interconnected, which makes it hard to single out one of those as the decisive one in shaping the spectrum. In this section, the possible contribution of the different factors to the observed trends is investigated. The connection with the $\mathrm{PAH} /$ dust ratio is also highlighted.

\subsubsection{Ionization balance}

The PAH charge is known to influence the PAH spectrum remarkably. Upon ionization, the intrinsic strength of the features in the $5-10 \mu \mathrm{m}$ region increases while the intrinsic strength of those in the $10-15 \mu \mathrm{m}$ region decreases (Langhoff 1996; Allamandola et al. 1999; Hudgins et al. 1999; Peeters et al. 2001b). The PAH charge is governed by the photoionization rate and electron recombination rate, and is therefore a function of the local physical conditions (Bakes \& Tielens 1994; Bakes et al. 2001), in particular the electron density and the strength of the FUV field illuminating the PDR (generally measured in units of the interstellar radiation field in the solar neighbourhood).

To investigate the possible role of the ionization balance in the observed trends, these two quantities have been determined for our sample sources. The Lyman continuum flux and the rms electron density of the $\mathrm{H}$ II regions were derived from radio measurements. For the LMC objects 
N4A, N11A, N83B and N159-5, this was based on new $6 \mathrm{~cm}$ radio data obtained with the Australia Compact Array radio telescope (Martín-Hernández et al. 2002, in prep.). For the Galactic sources, the radio data were taken from the literature when available (for an overview, see Martín-Hernández et al. 2001). The Lyman continuum flux was converted to solar luminosities using the models from Vacca et al. (1996). By using the radio sizes, the local radiation field is obtained expressed in units of $1.6 \times 10^{-6} \mathrm{~W} \mathrm{~cm}^{-2}$. A distance of $55 \mathrm{kpc}$ was adopted for the LMC, the distances of the Galactic sources are taken from Peeters et al. (2001a).

For 30 Doradus the estimate for the local radiation field is based on stellar population studies. Assumed is that all the radiation is emitted by $\mathrm{R} 136$, the stellar cluster at the core of 30 Doradus. The stellar content of R136 is taken from the stellar population list of Massey \& Hunter (1998). From this stellar list a total luminosity is derived by using the same stellar atmosphere models as for the other sample objects. The flux at the pointing position is then determined from the projected distance from the center of R136 to the ISOPHOT pointings. For the derivation of the rms electron density at our positions, a $6 \mathrm{~cm}$ radio map of 30 Doradus is used that was kindly provided to us by Lazendic et al. (in prep.) prior to publication. As a check on the results for the radiation field as determined from the stellar population study, the Lyman continuum has also been derived from these radio data. The two different results are in very good agreement.

The balance between neutral and ionized PAHs is governed by the ratio of the local radiation field and the electron density, $G /<n_{\mathrm{e}}>$. The Galactic H II regions are found to have a systematically lower $G /\left\langle n_{\mathrm{e}}\right\rangle$ than the Magellanic sources. However, no correlation is found between this parameter and any of the possible relative PAHfeature strengths. As an example, the relative $\mathrm{PAH}$-feature strength $I_{6.2} / I_{11.2}$ as a function of $G$ and $\left.G /<n_{\mathrm{e}}\right\rangle$ is shown in Fig. 5. We therefore conclude that the differences between the relative PAH-feature strengths cannot be attributed to the ionization balance of the PAHs. It is interesting to see that, although for the four 30 Dor positions $G /<n_{\mathrm{e}}>$ shows a reasonable variation, the $I_{6.2} / I_{11.2}$ ratio for these pointings is roughly the same.

To provide us with a further check on the local radiation field and also to get an additional constraint on the derived electron density, PDR diagnostic fine-structure line ratios have been determined from the SWS and LWS data of all our LMC objects (except N11A for which no LWS data is available - Vermeij et al. 2001) and all the IRAS sources (Peeters et al. 2001a). This is necessary because the PAH spectrum is formed in the PDR, whereas the physical parameters derived from the radio data are more indicative of the body of ionized gas making up the H II region.

The combination of the $[\mathrm{C}$ II $] 158 \mu \mathrm{m} /[\mathrm{O} \mathrm{I}] 63 \mu \mathrm{m}$ line ratio with the $([\mathrm{C}$ II $] 158+[\mathrm{OI}] 63+[\mathrm{Si} I \mathrm{II}] 35) / \mathrm{FIR}$ ratio defines a unique set of density and radiation field $G$ (e.g. Wolfire et al. 1990). It turns out that the Galactic
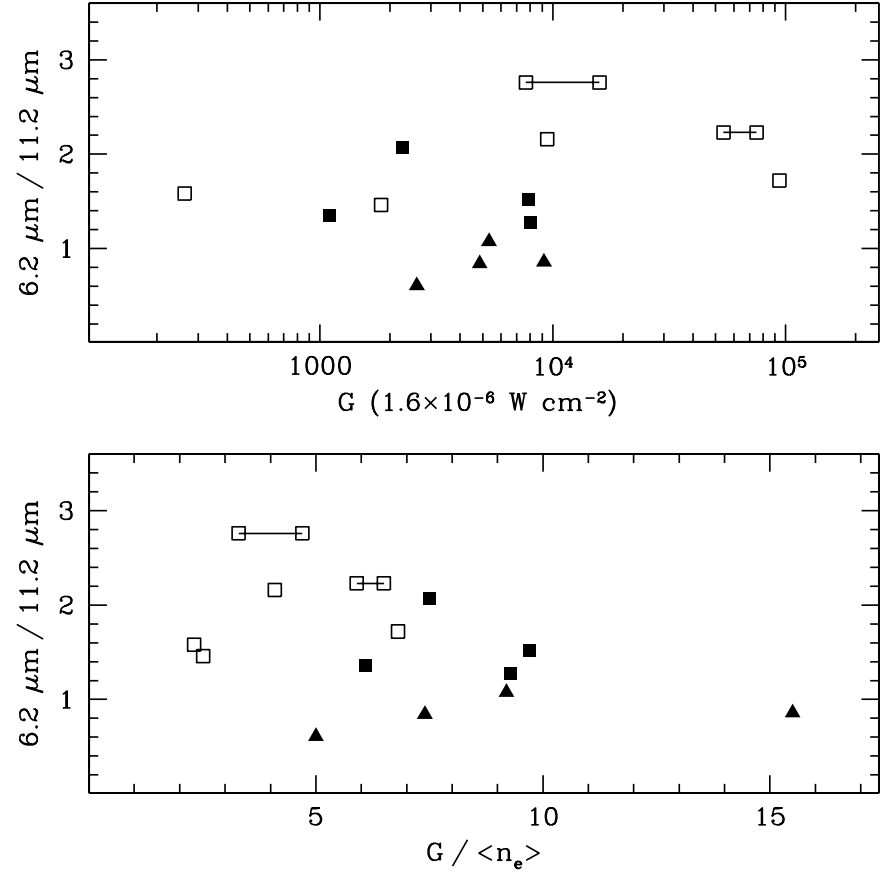

Fig. 5. The $I_{6.2} / I_{11.2}$ ratio versus the local radiation field $G$ and the parameter $G /<n_{\mathrm{e}}>$. The full lines join $G$ and $G /$ $\left.<n_{\mathrm{e}}\right\rangle$ as derived using both the near and far solar distance.

and Magellanic sources have similar diagnostic ratios. Our sources lie between the $\log G=3$ and $\log G=4$ tracks in the diagnostic diagrams from Wolfire et al. (1990), which is in reasonable agreement with our values of the local radiation field from the radio data. The LMC and Galactic H II regions show a similar efficiency of photoelectric heating as measured by the $([\mathrm{C}$ II $] 158+[\mathrm{O}$ I $] 63+[\mathrm{Si}$ II $] 35) / F I R$ ratio. The photoelectric effect is governed by the ionization state of the PAHs (Bakes \& Tielens 1994), so these findings lend further support to our conclusion that the ionization balance of the PAHs is not responsible for the observed differences in the PAH-feature strenghts.

\subsubsection{Dehydrogenation}

Theoretical studies of dehydrogenation of interstellar PAHs have shown that for PAHs larger than $\sim 25$ carbon atoms hydrogenation through reactions with abundant atomic hydrogen is more important than dehydrogenation through FUV photo-dissociation (Tielens et al. 1987; Allamandola et al. 1989; Jochims et al. 1994, 1999; Allain et al. 1996). With a typical PAH size of 50 C-atoms (Tielens et al. 2000), dehydrogenation should therefore be minimal and no effect on the PAH spectrum should be seen.

Observationally, the degree of dehydrogenation can be probed best by the features in the $10-15 \mu \mathrm{m}$ region. The peak position and strength of the $\mathrm{PAH}$ features present in this range depend on the structure of the molecule, in particular on the number of neighbouring $\mathrm{H}$-atoms per ring (e.g. Bellamy 1958; Hudgins \& Allamandola 1999; Hony et al. 2001). Aromatic rings carrying $\mathrm{CH}$ groups which 
have no neighbouring $\mathrm{CH}$ groups are termed "solo" $\mathrm{CH}$ groups, two adjacent $\mathrm{CH}$ groups "duet" $\mathrm{CH}$ groups, and so on. Other ways to assess the degree of dehydrogenation include a non-linear behaviour between the 3.3 and $11.2 \mu \mathrm{m}$ PAH features upon dehydrogenation (Hony et al. 2001) and the change in the ratio of the $\mathrm{CC}$ and $\mathrm{CH}$ modes.

In their observational study, Hony et al. (2001) conclude that dehydrogenation is unimportant for the objects in their sample, which includes the Galactic sources contained in ours. The LMC source with the smallest $I_{6.2} / I_{11.2}$ ratio for which there is also a reliable $3.3 \mu \mathrm{m}$ flux (30 Dor\#3), has a $I_{3.3} / I_{11.2}$ ratio that is consistent with those found in Hony et al. (2001) implying that the PAHs in this source are not dehydrogenated. This can unfortunately not be confirmed by its $10-15 \mu \mathrm{m}$ region since the $\mathrm{PAH}$ features there are very weak and no significant $12.7 \mu \mathrm{m}$ feature has been detected (Sturm et al. 2000, see Sect. 4.2.4). Nevertheless, our conclusion is that the effect of dehydrogenation cannot fully account for the observed differences in the PAH-feature strengths.

\subsubsection{Molecular size}

With decreasing size of the molecules, the ratio of $\mathrm{CC}$ to $\mathrm{CH}$ bonds also decreases. This $\mathrm{CC} / \mathrm{CH}$ ratio is most clearly reflected in the $I_{6.2} / I_{3.3}$ ratio, since the $3.3 \mu \mathrm{m}$ band is produced by all the $\mathrm{CH}$ bonds. The $3.3 \mu \mathrm{m}$ feature though is hardly detected in our ISOPHOT spectra, so we have to rely on the $I_{6.2} / I_{11.2}$ ratio.

Since the $11.2 \mu \mathrm{m}$ band is only produced by the solo $\mathrm{CH}$ groups, the $I_{6.2} / I_{11.2}$ ratio is influenced by both the molecular structure as well as the size. If we assume that there is only a size effect and restrict ourselves to PAHs with more than 50 carbon atoms, the lower $I_{6.2} / I_{11.2}$ ratio observed in 30 Doradus and the SMC would at first suggest that the PAHs are smaller than in the Galaxy and non30 Dor LMC sources (Schutte et al. 1993). However, the position with the lowest $I_{6.2} / \Sigma \mathrm{PAH}$ and $I_{6.2} / I_{11.2}$ ratio (30 Dor\#3) has a $I_{6.2} / I_{3.3}$ ratio that is consistent with the range observed for the Galactic H II regions. This would suggest that differences in molecular size are not responsible for the observed PAH-feature strengths.

\subsubsection{Molecular structure}

As was already mentioned in Sect. 4.2.2, the strength of the PAH features in the $10-15 \mu \mathrm{m}$ region depends on the number of neigbouring $\mathrm{CH}$ groups. The strength of the $11.2 \mu \mathrm{m}$ feature, and hence the $I_{6.2} / I_{11.2}$ ratio, is therefore influenced by the molecular structure. If the observed trend in this ratio were only due to the molecular structure, this would imply that the PAH emission in the SMC source and 30 Doradus is dominated by "smoothedged" species (the so-called "compact" species in Hony et al. 2001 which have long straight edges and few corners, cf. their Fig. 9) while open, "rough-edged" structures (the so-called open, uneven species in Hony et al. 2001 with

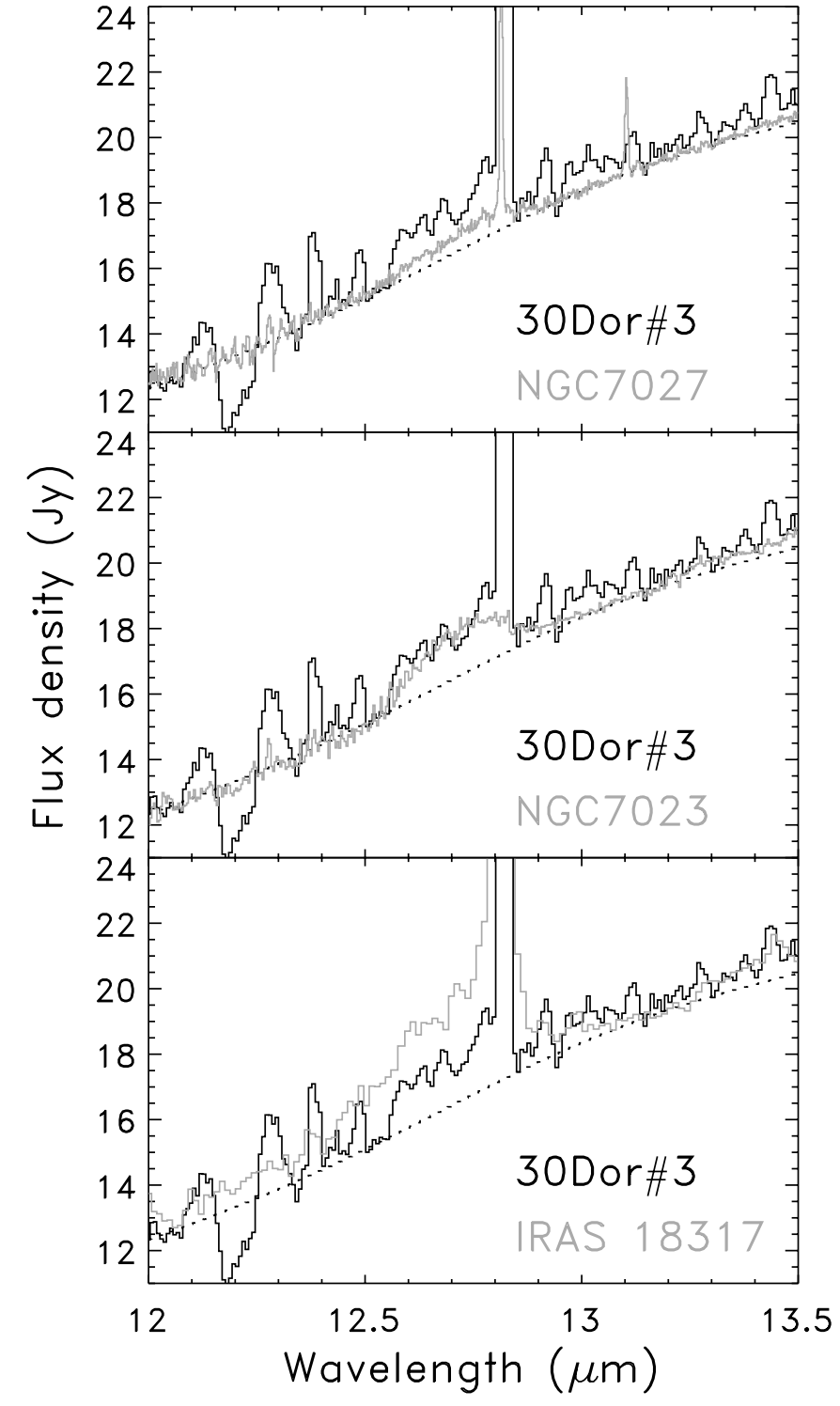

Fig. 6. A comparison between the $12.7 \mu \mathrm{m}$ feature of 30 Dor\#3 and the scaled $12.7 \mu \mathrm{m}$ feature of NGC 7027 (top), NGC 7023 (middle) and IRAS 18317 (bottom). The scaling is such that the $11.2 \mu \mathrm{m}$ feature is equally strong in all spectra. The 30 Dor \#3 spectrum is shown in solid black and the scaled spectra in grey. The dotted line represents the continuum.

many corners, cf. their Fig. 9) are the dominant ones in the Galactic and non-30 Doradus H II regions. To have a strong handle on this, the features in the $10-15 \mu \mathrm{m}$ spectral region are indispensible.

We carefully re-examined the spectrum of 30 Dor \#3. A hint of the $12.7 \mu \mathrm{m}$ feature is found, but it is clearly no $3 \sigma$ detection. In order to get a feeling for the $I_{12.7} / I_{11.2}$ ratio, we took the spectra from Hony et al. (2001) with the lowest and highest $I_{12.7} / I_{11.2}$ ratio and scaled them in such a way that their $11.2 \mu \mathrm{m}$ feature is as strong as in the 30 Dor\#3 spectrum (see Fig. 6). It is obvious from Fig. 6 that for the Galactic source with the highest $I_{12.7} / I_{11.2}$ ratio (IRAS 18317), the scaled $12.7 \mu \mathrm{m}$ feature cannot be hidden in the noise of the spectrum of 30 Dor\#3. The same 
is not true, however, for the Galactic source with the lowest $I_{12.7} / I_{11.2}$ ratio (NGC 7027). The source with a similar $I_{12.7} / I_{11.2}$ ratio as 30 Dor $\# 3$, is the reflection nebula NGC 7023, which is intermediate in the range of $I_{12.7} / I_{11.2}$ ratios studied by Hony et al. (2001). We can therefore conclude that the PAHs in 30 Dor \#3 are certainly more compact when compared to those in the Galactic H II region IRAS 18317 and are probably similar to those in the reflection nebula NGC 7023.

The SMC B1\#1 molecular cloud strongly exhibits a $12.7 \mu \mathrm{m}$ feature (Reach et al. 2000). The observed $I_{12.7} / I_{11.2}$ and $I_{6.2} / I_{11.2}$ ratios for this source also point towards species that are more compact compared to those in the Galactic H II regions (Hony et al. 2001).

To summarize, the few observations present to date point towards the dominance of compact $\mathrm{PAH}$ species in the SMC and 30 Doradus while open, uneven structures are the dominant ones in Galactic HII regions and the non-30 Dor LMC sources.

\subsubsection{PAH/dust abundance}

An indicator of the $\mathrm{PAH} /$ dust abundance is the ratio between the total emission in the PAHs and the far-infrared (FIR) emission. We derived the FIR emission from LWS spectra of the objects (Vermeij et al. 2001; Peeters et al. 2001a). The $\Sigma$ PAH / FIR ratios are very similar for the Galactic and LMC sources except that the four pointings towards 30 Doradus show lower values. However, the LWS aperture is much larger compared to the ISOPHOT and ISO-SWS apertures. This ratio will therefore be influenced by the aperture differences for sources more extended then the mid-IR beam or for sources which are not isolated. It is quite certain that the mid-IR and FIR beams receive radiation from different regions in 30 Doradus. After correcting for the aperture difference (assuming a uniform, infinitely extended source), the $\Sigma \mathrm{PAH} / \mathrm{FIR}$ ratios for the 30 Doradus observations are similar to the ratio of the other sources. Note, however, that some of the other sources are not isolated or are more extended then the mid-IR beam (but certainly smaller then the FIR beam) and could therefore suffer from the same problem.

If we assume that all the UV radiation absorbed by the dust is re-emitted in the IR, the $\Sigma \mathrm{PAH} / G$ ratio is an additional indicator for the $\mathrm{PAH} /$ dust abundance. As can be seen in Fig. 7, the $\mathrm{PAH} / G$ ratio in the LMC lies at the lower end of the range covered by our sample H II regions. Both the 30 Doradus and non-30 Doradus observations have a similar $\Sigma \mathrm{PAH} / G$ ratio. The PAH/dust abundance thus appears to be very similar for the LMC sources. However, the difference in the $\Sigma \mathrm{PAH} / G$ ratio between the Magellanic Cloud and Galactic sources seems significant.

It is currently believed that PAHs form either as byproducts or intermediares in soot formation (Frenklach \& Freigelson 1989; Cherchneff et al. 1992) or as shattering products of grains (Jones et al. 1996). Apparently, the differences in metallicity and general conditions in the

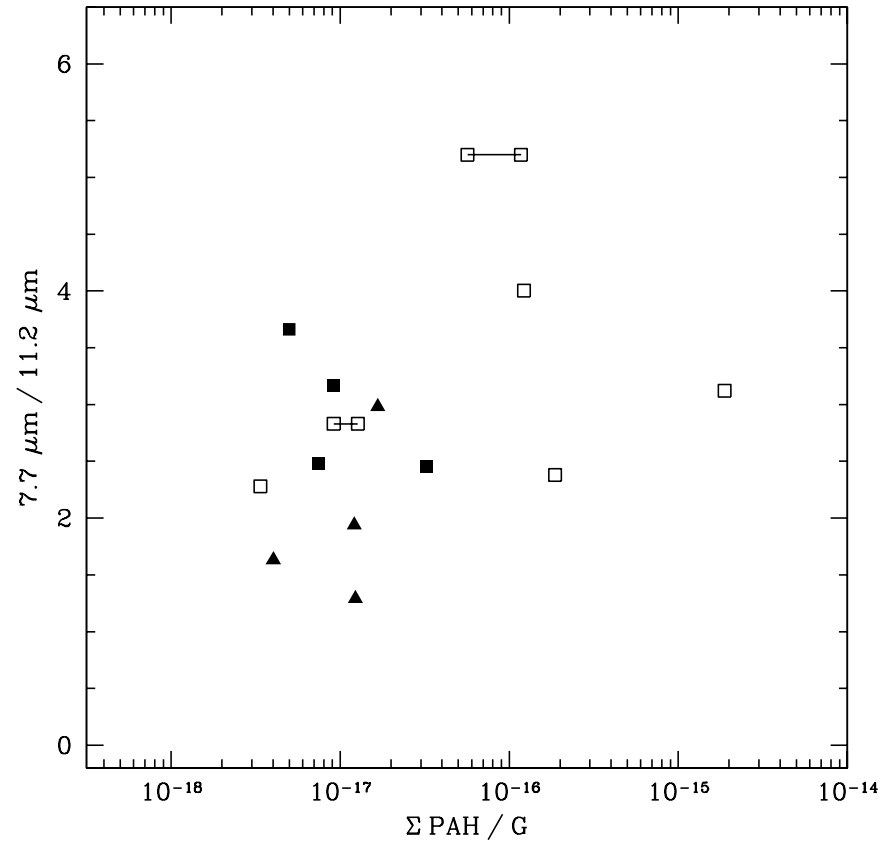

Fig. 7. The correlation between the $I_{7.7} / I_{11.2}$ ratio and the $\Sigma \mathrm{PAH} / G$ ratio. The full lines join the $\Sigma \mathrm{PAH} / G$ as derived using both the near and far solar distance.

ISM or in soot-forming regions have some influence on the $\mathrm{PAH} /$ dust ratio.

\section{Summary}

In this paper a set of ISOPHOT spectra from HII regions in the Large Magellanic Cloud is presented. In all the spectra, emission bands arising from Polycyclic Aromatic Hydrocarbons (PAHs) are clearly present. These features are observed to vary considerably in relative strength to each other from source to source and even within 30Dor. The LMC spectra have been compared with ISO-SWS spectra from Galactic H II regions and with the ISOCAM observation towards a quiescent molecular cloud in the SMC (Reach et al. 2000).

A correlation is found between the $I_{7.7} / I_{11.2}$ versus $I_{6.2} / I_{11.2}$ and the $I_{8.6} / I_{11.2}$ versus $I_{6.2} / I_{11.2}$ ratios. A segregation between the sources of different type of environment, i.e. from the Milky Way to the LMC and to the $\mathrm{SMC}$, is present. Furthermore, within the LMC observations, a clear distinction between 30 Doradus and non30 Doradus pointings is found. So, the relative strength of the IR emission features correlate with the local environment.

A similar sequence can be found for the $2175 \AA$ bump strength, which decreases in strength from the Milky Way to the non-30 Dor pointings, to the 30 Dor pointings and is even completely absent in the SMC (e.g. Rodrigues et al. 1997). Since the lines-of-sight for the extinction derivation and for the ISOPHOT observations are very similar, this suggests that probably the same conditions responsible for the observed trends in the relative $\mathrm{PAH}$-feature strengths 
also affect the carrier of the $2175 \AA$ bump leading to the differences in strength of the latter.

We have discussed the different factors that influence the spectra and might explain the observed variations. Most important of these factors is the ionization balance of the PAHs which shows no difference between the Magellanic and Galactic sources. The $\mathrm{PAH} / G$ ratio, which is an indicator of the $\mathrm{PAH} /$ dust abundance, appears to be very similar for the LMC sources, but the difference in the $\Sigma \mathrm{PAH} / G$ ratio between the Magellanic Cloud and Galactic sources seems significant. The differences in metallicity and general conditions in the ISM or in soot-forming regions have apparently some influence on the $\mathrm{PAH} /$ dust ratio.

The few observations present to date point towards the molecular structure as the major cause of the observed variations. Compact PAH species dominate in the SMC and in 30 Doradus, while PAH species with an open, uneven structure are the dominant ones in Galactic H II regions and the non-30 Dor LMC sources. This may reflect the effects of the local physical conditions on the composition and characteristics of the PAH family.

Acknowledgements. PIA is a joint development by the ESA Astrophysics Division and the ISO-PHT consortium. EP acknowledges the support from an NWO program subsidy (grant number 783-70-000).

\section{References}

Acosta-Pulido, J. A., \& Ábrahám, P. 2001 ESA SP-481, The calibration legacy of the ISO mission, ed. L. Metcalfe, \& M. F. K. Kessler, in press

Allain, T., Leach, S., \& Sedlmayr, E. 1996, A\&A, 305, 616

Allamandola, L. J., Tielens, A. G. G. M., \& Barker, J. R. 1985, ApJ, 290, L25

Allamandola, L. J., Tielens, A. G. G. M., \& Barker, J. R. 1989, ApJS, 71, 733

Allamandola, L. J., Hudgins, D. M., \& Sandford, S. A. 1999, ApJ, 511, L115

Bakes, E. L. O., \& Tielens, A. G. G. M. 1994, ApJ, 427, 822

Bakes, E. L. O., Tielens, A. G. G. M., \& Bauschlicher, C. M. 2001, ApJ, 556, 501

Bellamy, L. 1958, in The infrared spectra of complex molecules 2nd edition (John Wiley \& Sons, Inc., New York)

Borgman, J., van Duinen, R. J., \& Koorneef, J. 1975, A\&A, 40, 461

Bosselli, A., et al. 1997, A\&A, 324, L13

Cesarsky, D., Jones, A. P., Lequeux, J., \& Verstraete, L. 2000, A\&A, 358, 708

Clayton, G. C., \& Martin, P. G. 1985, ApJ, 288, 558

Cherchneff, I., Barker, J. R., \& Tielens, A. G. G. M. 1992, ApJ, 401, 269

Cohen, M., Dopita, M. A., \& Schwartz, R. 1986, ApJ, 302, 737

Cohen, M., Tielens, A. G. G. M., Bregman, J., et al. 1989, ApJ, 341,246

Cole, A. A., Nordsieck, K. H., Gibson, S. J., \& Harris, W. M. 1999, AJ, 118, 2280

Contursi, A., Lequex, J., Hanus, M., et al. 1998, A\&A, 336, 662

Contursi, A., Lequex, J., Cesarsky, D., et al. 2000, A\&A, 362, 310
Fitzpatrick, E. L. 1985, ApJ, 299, 219

Fitzpatrick, E. L. 1986, AJ, 92, 1068

Frenklach, M., \& Feigelson, E. D. 1989, ApJ, 341, 372

Geballe, T. R., Lacy, J. H., Persson, S. E., et al. 1985, ApJ, 292,500

Gillett, F. C., Forrest, W. J., \& Merrill, K. M. 1973, ApJ, 183,87

Helou, G. 1999, ESA SP-427 The Universe as seen by ISO, ed. P. Cox, 727

Henize, K. G. 1956, ApJS, 2, 315

Hony, S., Van Kerckhoven, C., Peeters, E., et al. 2001, A\&A, 370, 1030

Hudgins, D. M., \& Allamandola, L. J. 1999, ApJ, 516, L41

Joblin, C., Léger, A., \& Martin, P. 1992, ApJ, 393, 79

Jochims, H. W., Ruhl, E., Baumgärtel, H., Tobita, S., \& Leach, S. 1994, ApJ, 420, 307

Jochims, H. W., Baumgärtel, H., \& Leach, S. 1999, ApJ, 512, 500

Jones, A. P., Tielens, A. G. G. M., \& Hollenbach, D. J. 1996, ApJ, 469, 740

Koornneef, J., \& Code, A. D. 1981, ApJ, 247, 860

Langhoff, S. R. 1996, J. Phys. Chem. A, 100, 2819

Léger, A., \& Puget, J. L. 1984, A\&A, 137, L5

Lutz, D., Spoon, H. W. W., Rigopoulou, D., et al. 1998, ApJ, 505, L103

Martín-Hernández, N. L., Peeters, E., Morisset, C., et al. 2001, $\mathrm{A} \& \mathrm{~A}$, in press

Massey, P., \& Hunter, D. A. 1998, ApJ, 493, 180

Moutou, C., Verstraete, L., Sellgren, K., \& Léger, A. 1999, ESA SP-427, The Universe as seen by ISO, ed. P. Cox, 727

Nandy, K., Morgan, D. H., Willis, A. J., Wilson, R., \& Gondhalekar, P. M. 1981, MNRAS, 196, 955

Pauzat, F., Talbi, D., \& Ellinger, Y. 1997, A\&A, 319, 318

Peeters, E., Martín-Hernández, N. L., Damour, F., et al. 2001a, $\mathrm{A} \& \mathrm{~A}$, in press

Peeters, E., Hony, S., Van Kerckhoven, C., et al. 2001b, submitted

Prévot, M. L., Lequeux, J., Maurice, E., Prévot, L., \& RoccaVolmerange, B. 1984, A\&A, 132, 389

Puget, J. L., \& Léger, A. 1989, ARA\&A, 27, 161

Reach, W. T., Boulanger, F., Contursi, A., \& Lequeux, J. 2000, A\&A, 361, 895

Roche, P. F., Aitken, D. K., \& Smith, C. H. 1989, MNRAS, 236,485

Rodrigues, C. V., Magalhães, A. M., Coyne, G. V., \& Piirola, V. 1997, ApJ, 485, 618

Schutte, W. A., Tielens, A. G. G. M., \& Allamandola, L. J. 1993, ApJ, 415, 397

Sturm, E., Lutz, D., Tran, D., et al. 2000, A\&A, 358, 481

Tielens, A. G. G. M., Allamandola, L. J., Barker, J. R., \& Cohen, M. J. 1987, in Polycyclic Aromatic Hydrocarbons and Astrophysics, 273

Tielens, A. G. G. M., Van Kerckhoven, C., Peeters, E., \& Hony, S. 2000, IAUS, 197, 349

Vacca, W. D., Garmany, C. D., \& Shull, J. M. 1996, ApJ, 460, 914

Vermeij, R., Damour, F., Van der Hulst, J. M., \& Baluteau, J.-P. 2001, A\&A, submitted

Verstraete, L., Puget, J. L., Falgarone, E., et al. 1996, A\&A, 315, L337

Wolfire, M. G., Tielens, A. G. G. M., \& Hollenbach, D. 1990, ApJ, 358, 116 